7-17-2020

\title{
JMASM 53: MicceriRD
}

Michael Lance

ExceLance, LLC, michael.lance@gmail.com

Follow this and additional works at: https://digitalcommons.wayne.edu/jmasm

Part of the Applied Statistics Commons, Social and Behavioral Sciences Commons, and the Statistical Theory Commons

\section{Recommended Citation}

Lance, M. (2019). JMASM 53: MicceriRD. Journal of Modern Applied Statistical Methods, 18(2), eP3391. doi: $10.22237 /$ jmasm/1604190720

This Algorithms and Code is brought to you for free and open access by the Open Access Journals at DigitalCommons@WayneState. It has been accepted for inclusion in Journal of Modern Applied Statistical Methods by an authorized editor of DigitalCommons@WayneState. 


\section{JMASM 53: MicceriRD}

\section{Michael Lance}

ExceLance, LLC

Plymouth, MI

Fortran 77 and 90 modules (REALPOPS.lib) exist for invoking the 8 distributions estimated by Micceri (1989). These respective modules were created by Sawilowsky et al. (1990) and Sawilowsky and Fahoome (2003). The MicceriRD (Micceri's Real Distributions) Python package was created because Python is increasingly used for data analysis and, in some cases, Monte Carlo simulations.

Keywords: $\quad$ Micceri, real distributions, Python, Monte Carlo simulation, robustness

In 1989, Theodore Micceri published "The Unicorn, the Normal Curve, and Other Improbable Creatures" with the purpose of illustrating the variety of real distributions found in real datasets that are often not normally-distributed. His article had important implications for the use of statistical procedures on nonnormal datasets. When simulating data in the "real world", it helps to sample from such distributions. Thus, REALPOPS.lib was created by Sawilowsky et al. (1990) and Sawilowsky and Fahoome (2003) for this very purpose. Using these, many subsequent articles have been published on what happens when data are not normal and parametric tests are used (and how accuracy and power can be improved when using non-parametric tests on data from such distributions, even with small samples).

The MicceriRD (Micceri's Real Distributions) Python package was created because Python is increasingly used for data analysis and, in some cases, Monte Carlo simulations. Arrays of samples are generated in order to produce arrays of results as this is much more efficient than generating a sample and result within each nested loop of a Monte Carlo simulation. The random number generator used in drawing samples via NumPy is from the Parallel Congruent Generator (SciPy Community, 2019) family (PCG XSL RR 128/64), which has a period of $2^{126}$ (O’Neill, 2014).

doi: 10.22237/jmasm/1604190720 | Accepted: January 28, 2020; Published: July 17, 2020.

Correspondence: Michael Lance, michael.lance@gmail.com 


\section{MICHAEL LANCE}

MicceriRD has the following functions for each of Micceri's 8 real distributions:

1. It invokes each distribution by name as a NumPy array.

2. It draws a NumPy array of $k$ random samples of $n$ size from each distribution, by name.

\section{Sample Code}

Installation from command prompt:

pip install MicceriRD

(current version at the time of this writing: 0.2)

Invoke the Digit Preference distribution as a NumPy array:

In[1]: import MicceriRD as mrd

In[2]: mrd.DigitPreference

Out [2]: $\operatorname{array}([420,420,425, \ldots, 635,635,635])$

Draw 4 random samples of $n=6$ as a NumPy array from the Digit Preference distribution:

In[3]: mrd.Sampling.DigitPreference $(4,6)$

Out [3]:

$\operatorname{array}([[520,460,515,465,525,560]$,

$[585,575,585,570,475,470]$,

$[470,550,475,580,500,590]$,

$[525,595,535,515,525,470]])$

MicceriRD on the Python Package Index:

https://pypi.org/project/MicceriRD/

MicceriRD homepage:

https://bitbucket.org/mikeronni/miccerird/

\section{References}

Micceri, T. (1989). The unicorn, the normal curve, and other improbable creatures. Psychological Bulletin, 105(1), 156-166. doi: 10.1037/0033-

2909.105.1.156 


\section{MICCERIRD}

O’Neill, M. E. (2014, September 5). PCG: A family of simple fast spaceefficient statistically good algorithms for random number generation (Technical report no. HMC-CS-2014-0905). Claremont, CA: Harvey Mudd College Computer Science Department. Retrieved from https://www.cs.hmc.edu/tr/hmccs-2014-0905.pdf

Sawilowsky, S. S., Blair, R. C., \& Micceri, T. (1990). REALPOPS.LIB: APC Fortran library of eight real distributions in psychology and education. Psychometrika, 55(4), 729.

Sawilowsky, S. S., \& Fahoome, G. F. (2003). Statistics via Monte Carlo simulation with Fortran. Rochester Hills, MI: JMASM.

SciPy Community. (2019, July 26). Parallel congruent generator (64-bit, PCG64) [NumPy routine]. Retrieved from

https://docs.scipy.org/doc/numpy/reference/random/bit_generators/pcg64.html 